\title{
Sphenochoanal Polyp in a Young Adult Female Presenting with Epistaxis
}

\author{
Arup Sen Gupta ${ }^{1 *}$, Aloke Bose Majumdar ${ }^{1}$, Ranabir Pal ${ }^{2}$ and Shrayan Pal ${ }^{2}$
}

${ }^{1}$ Department of Otorhinolaryngology, MGM Medical College and LSK Hospital, Kishanganj, Bihar-855107, India

${ }^{2}$ Sikkim Manipal Institute of Medical Sciences (SMIMS) and Central Referral Hospital (CRH) 5th Mile, Tadong, Gangtok, Sikkim-737 102, India

\begin{abstract}
Sphenochoanal polyp is a rare entity which originates from the sphenoid sinus and passes via the sphenoid ostium to the choana and presents like the more frequently occurring antrochoanal polyp. The authors reported the case of a 22-year-old woman who was diagnosed with a left sphenochoanal polyp that is a relatively uncommon entity. If unrecognized they can be mistaken for an antrochoanal polyp for which longer the delay in treatment more are the chances of complications. Pre-operative investigations included axial and coronal computed tomography. The sphenochoanal polyp was effectively treated using an endoscopic sinus surgery. Here the case is presented along with a review of the literature. To the horizon of our knowledge, no previous case of sphenochoanal polyp was reported from Eastern India. In conclusion we have to introduce patient education for the early detection of polyps and their interventions.
\end{abstract}

Keywords: Polyp; Sphenochoanal sphenoid sinus; Choanal

\section{Introduction}

Isolated polyp originate from the maxillary, ethmoids or sphenoid sinuses and pass via the ostium into the nasal cavity and posteriorly into the choana presenting as choanal polyp. Though mostly the polyps arise in the maxillary sinus, but in rare cases polyps arise from the sphenoid sinus and extending to the choana with uncertain etiology. These sphenochoanal polyps crop up most often in adolescents and young adults as the rare lesion originating from the sphenoid sinus mucosa and emerging from the sphenoidal ostium to land up in the nasopharynx. They are usually misdiagnosed for antrochoanal polyp that occurs more frequently with symptoms of nasal obstruction, nasal discharge and mouth breathing; headache and otalgia may also be a presenting feature. The presence of a choanal polyp in an atypical location can lead to diagnostic confusion and exploration of the wrong sinus and which will lead to recurrence. Due to the presence of important structures adjacent to the sphenoid sinus, an appropriate pre-operative nasal endoscopic evaluation is mandatory, along with the neurological and ophthalmological assessments. Computerized tomography of nose and sinus is the standard radiological diagnostic procedure to identify the site of origin of the polyp and plan the operative intervention. A proper diagnosis and surgical treatment are the basis to prevent recurrence. Endoscopic removal is suitable for all ages and is associated with low morbidity comprehensive elimination of the polyp, including its site of origin that minimizes the risk of recurrence. Early removal and long term follow up of sphenochoanal polyps usually prevent complication. The safety and efficacy of endoscopic removal of sphenochoanal polyp has been reported by researchers from different parts of the world [16]. The present study reported the case of a 22-year-old woman who was diagnosed with an isolated polyp arising from the sphenoid sinus.

\section{Case Report}

A 22 year old Muslim female presented with a history of recurrent epistaxis, headache, progressive nasal obstruction and nasal discharge with occasional bouts of chocking during swallowing. The patient was appraised with routine anterior and posterior rhinoscopy and computed tomography of the paranasal sinuses. The polyp completely blocked the choana on swallowing. Rest of the otorhinolaryngological examination showed no additional noteworthy abnormality. Preliminary investigations included axial and coronal computed tomography of the nose and sinuses that revealed a polypoidal mass arising from the left sphenoid sinus extending via the posterior part of nasal cavity into the choana. Other paranasal sinuses were within normal limits of evaluations. Detailed nasal endoscopy was done preoperatively to confirm the interim diagnosis of sphenochoanal polyp as suspected from computerized tomography (CT) scan. The examination revealed a smooth relatively insensitive to touch polypoidal mass seen in the (Figure 1) posterior part of nasal cavity that was extending into the choana and filling almost whole of the choana; middle meatus was relatively morbidity free. The polyp completely blocked the choana on swallowing.

The pre-operative planning was in favour of an endoscopic sinus surgery approach under general anaesthesia. Preoperative decongestion of the nasal cavity was contemplated with lignocaine and adrenaline soaked cotton pledgets prior to the surgical intervention for convenient approach of the polyp located at the posterior part of the nasal cavity.

A high risk informed consent was taken after counseling the patient for the procedure explaining in detail the risks with the advantages

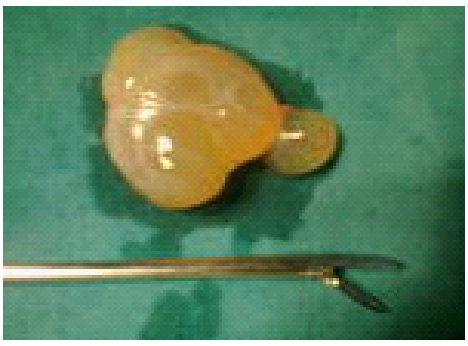

Figure 1: Polypoidal tissue delivered from nasal cavity and sphenoid sinus.

${ }^{*}$ Corresponding author: Dr. Arup Sen Gupta, Associate Professor, Departmen of Otorhinolaryngology, MGM Medical College and LSK Hospital, Kishanganj, Bihar-855107, India, Tel: +91-0353 2515962; Fax: +91-0353 2517854; E-mail: drarupsengupta@yahoo.co.in

Received June 02, 2012; Accepted July 09, 2012; Published July 11, 2012

Citation: Gupta AS, Majumdar AB, Pal R, Pal S (2012) Sphenochoanal Polyp in a Young Adult Female Presenting with Epistaxis. J Clin Case Rep 2:169. doi:10.4172/2165-7920.1000169

Copyright: ( 2012 Gupta AS, et al. This is an open-access article distributed under the terms of the Creative Commons Attribution License, which permits unrestricted use, distribution, and reproduction in any medium, provided the original author and source are credited. 
and disadvantages involved in anesthesia and the course of actions of further interventions. In the preliminary history and pre-operative clinical examination there was no disadvantage to the patient for the proposed intervention under general anesthesia. The middle and superior turbinates and superior part of the nasal septum adjacent to these turbinates were meticulously injected with 1: 1,00,000 lignocaine in adrenaline solution for further decongestion of the surgical field. After a period of ten minutes of further packing the area with lignocaine and adrenaline packs the middle and superior turbinates were gently lateralized to create working space for approaching the stalk of the polyp.

In the endoscopic view (Figure 2), the polyp was seen arising from within the cavity of the left sphenoid sinus. The stalk of the polyp was truncated at the ostium and removed without significant bleeding and by tracing the stalk proximally we were able to confirm the exit through the ostium of sphenoid sinus. Sphenoidal ostium was enlarged in medial and inferior direction to avoid injury to optic nerve and carotid artery with a narrow Kerrison's punch and on entering into the sphenoidal cavity the stalk was seen arising from the floor of sphenoid sinus which was first felt with a ball probe and then removed completely with the through cutting forceps without damaging the surrounding sphenoid mucosa or the inter-sphenoid septum. In the post operative period the women recovered uneventfully.

The specimen was macroscopically measured to be of the dimensions of $5 \times 2.5 \mathrm{~cm}$. The specimen was immediately sent for histo-pathological investigations that revealed of an inflammatory polyp, composed of loose mucoid stroma and mucous glands covered by respiratory epithelium. There were reported goblet cell hyperplasia and cellular infiltrates consisting of lymphocytes, eosinophil and plasma cells.

Follow-up evaluations were made at every month and even at the end of a year the patient had most favourable post-operative outcomes. Further, the patient showed no recurrence and was free of all the complaints which she previously had. The institutional authority approved the publication of this report.

\section{Discussion}

The lady presented with nasal obstruction and headache that was chronically reported to the local doctor during epistaxis for which she was referred to our centre. Choanal polyps most commonly arise from maxillary sinus followed by sphenoid sinus and rarely from ethmoidal sinus. There are no reports of choanal polyps from frontal sinus. Most cases reported were during the past decade.

Benign intramural cysts were found in four percent of normal asymptomatic population that were precursors of choanal polyps. Spraggs [7] reported a case of solitary mass arising from the sphenoid sinus extending through the sphenoid ostium into the sphenoethmoidal recess and choana which on tomographic scan showed (Figure 3) as a solitary mass of low attenuation. Crampette et al. [8] reported two cases of sphenochoanal polyps in children. Further, Eloy et al. [9] stressed the importance of endoscopic surgery in two cases of sphenochoanal polyps. Gordts et al. [10] also reported four unusual cases of sphenochoanal polyps. Illeri et al. [11] reported a case of large sphenochoanal polyp.

Ethmoid sinus and sphenoid sinus are unusual sites for origin of the choanal polyps apart from typical site of maxillary sinus. Vascular

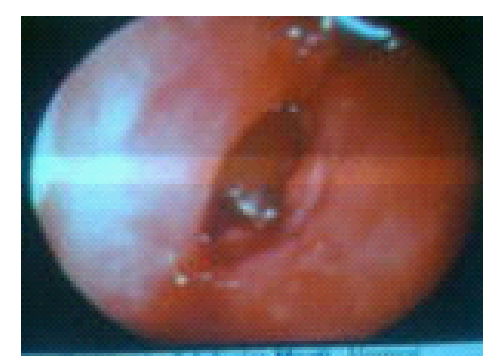

Figure 2: Endoscopic view of enlarged sphenoid sinus opening.

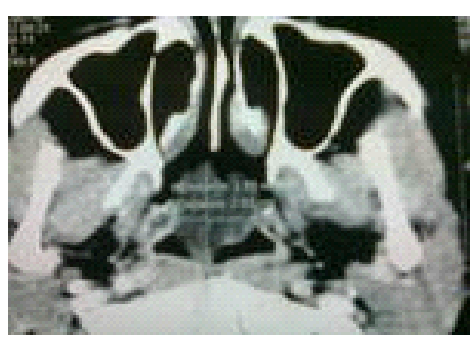

Figure 3: Computed tomographic view of paranasal sinuses showing polyp extending in choana.

changes in choanal polyps make them difficult to differentiate from vascular neoplasms. The clinical, nasal and nasopharyngeal endoscopic examination and CT scans are important for the diagnosis of sphenochoanal polyps. CT scans correctly demonstrate (Figure 4) the origin of choanal polyps and eliminates the risk of opening the wrong sinus. Maldonaldo et al. [6] mentioned the need for CT scan for definitive diagnosis of sphenochoanal polyps however occasional occupation of the maxillary sinus with mucous can mimic a polyp which needs to be differentiated by MRI studies with gadolinium contrast. CT scan shows choanal polyps as hypo-attenuating lesions with clear indication of sinus of origin. The relation of the choanal polyp to the middle turbinate is an important differentiating point between polyps of antral origin as compared to the sphenochoanal polyps which are medial to the middle turbinate. The ostium of the sinus is usually widened due to long standing compression of polyp which is also helpful to determine the origin of the polyp [12,13]. Radioallergosorbent Test (RAST) test for specific allergens and skin prick tests show no correlation of choanal polyps with allergy [14]. Berg et al. [12] demonstrated similar protein characteristics between fluid aspirates from choanal polyps and intramural cysts. Choanal polyps do not differ histologically from simple nasal polyps. Berg demonstrated similar protein characterstics between fluid aspirates from choanal polyps and intramural cysts. Min et al. [13] demonstrated the eosinophillic infiltration was less pronounced in antrochoanal polyps than in allergic polyps and the presence of submucosal glands was less pronounced [15]. According to a report by Zucrkand the number of cases reported in the literature is small and the most common symptom being nasal obstruction.

We are yet to find any report on the explorative study on the effectiveness of medical therapy of sphenochoanal polyps. In the present state of development of intervention, endoscopic removal of sphenochoanal polyps with complete intra sinusal clearance is the most effective treatment as opposed to avulsion which has a sequel of high recurrence. Soh et al. [16] emphasized the use of microdebrider for debulking the polyp then removing the intrasinusal part completely 


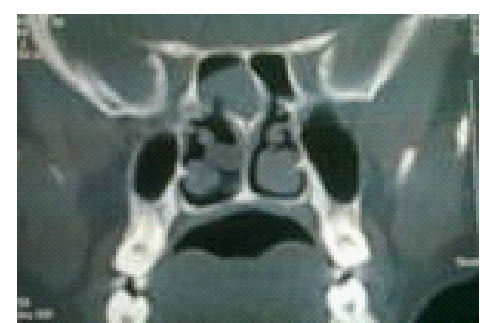

Figure 4: CT view of nasal cavity and sinuses demonstrating soft tissue opacity in left sphenoid sinus.

after widening the ostium. The microdebrider is the most effective instrument to debulk and completely remove the sphenochoanal polyp. Dadas et al. [15] reported a case of sphenochoanal polyp which was successfully removed by partial removal of anterior wall of the sphenoid sinus after a sphenoidotomy and complete removal of polyp. Penavic et al. [14] reported a case of sphenochoanal polyp in a 19 years old male patient which was successfully removed by powered instrument under endoscopic control. Tysome et al. [4] reported a case of sphenochoanal polyp with concomitant nasal polyp in a 54 years old male patient presenting with bilateral nasal obstruction, sleep apnoea and altered voice which was successfully removed under local anesthesia as the patient suffered from cardiomyopathy and was unfit for general anesthesia. Nayak et al. [5] reported a case of sphenochoanal polyp with heterotrophic glial tissue.

Primary outcome measure was successful removal of polyp by endoscopic sinus surgery as the utmost priority to prevent known secondary complications of the choanal polyps. In treating a choanal polyp of sphenoidal origin it is ideal to apply endoscopic sinus techniques for their successful removal. In our case the sphenoidal polyp originated from the floor of the left sphenoid sinus which after excising the ostial part and subsequently performing a sphenoidotomy to enlarge the natural ostium for better access the intra sinusal root of the polyp could be successfully removed without any trauma to the adjacent vital structures.

The strength of the study is that the case had undergone optimum management within the resources constraints setup of an eastern Indian tertiary care hospital. Further the case has been reported with the precise details including the follow-up. To the horizon of our knowledge, no previous study has been reported of sphenochoanal polyps in eastern India. We had the limitation of reporting the case little late than expected.

Further research directions should move around research collaboration in this part of the country for sphenochoanal polyps. The general population needs to be educated on the early identification of polyps and the optimum interventions is by prompt endoscopic removal of sphenochoanal and other polyps under general anesthesia as the safe and effective method. Training of primary health care staffs with clinical audit are needed for handling these emergencies without referring them to other centers can save morbidity with long term complications. Adequate preoperative evaluation of computed tomography and detailed nasal endoscopy is mandatory to ascertain the correct diagnosis and facilitate the planning of appropriate surgical procedure avoiding unnecessary exploration of maxillary sinus.

\section{References}

1. Al-Qudah MA (2010) Sphenochoanal polyp: current diagnosis and management. Ear Nose Throat J 89: 311-317.
2. Altun H, Teker AM, Ceran M, Gedikli O (2008) [Endoscopic approach in patients with choanal polyps]. Kulak Burun Bogaz Ihtis Derg 18: 74-78.

3. Nour YA, Al-Madani A, El-Daly A, Gaafar A (2008) Isolated sphenoid sinus pathology: spectrum of diagnostic and treatment modalities. Auris Nasus Larynx 35: 500-508.

4. Tysome JR, Saleh HA (2007) Sphenochoanal polyp presenting with concomitant nasal polyps. Ear Nose Throat J 86: 50-52.

5. Nayak DR, Pujary K, Valiathan M, Parul P, Kamat A (2007) Sphenochoana polyp with heterotopic glial tissue. J Laryngol Otol 121: 274-276.

6. Maldonado M, Alobid I, Bernal-Sprekelsen M, Mullol J (2006) [Sphenochoanal polyp. Diagnostic and therapeutic aspects]. Acta Otorrinolaringol Esp 57: 149151.

7. Spraggs PD (1993) Radiological diagnosis of spheno-choanal polyp. J Laryngol Otol 107: 159-160

8. Crampette L, Mondain M, Rombaux P (1995) Sphenochoanal polyp in children Diagnosis and treatment. Rhinology 33: 43-45.

9. Eloy P, Evrard I, Bertrand B, Delos M (1996) Choanal polyp of sphenoida origin. Report of two cases. Acta Otolaryngol Belg 50: 183-189.

10. Gordts F, Clement PA (1997) Unusual choanal polyps. Acta Otolaryngol Belg 51: $177-180$.

11. lleri F, Koybasioglu A, Uslu S (1998) Clinical presentation of spenochoanal polyp. Eur Arch Otorhinolaryngol 255: 138-139.

12. Berg O, Carenfelt C, Silfversward C, Sobin A (1998) Origin of the choana polyp. Arch Otolaryngol Head Neck Surg 114: 1270-1271.

13. Min YG, Chung JW, Shin JS, Chi JG (1995) Histologic structure of antrochoanal polyps. Acta Otolaryngol 115: 543-547.

14. Pajić-Penavić I, Đanić D, Fuštar-Preradović L (2009) Sphenochoanal polyposis Medicinski Glasnik 6: 271-273.

15. Dadas B, Yilmanz O, Vural C, Calis AB, Turgut S (2000) Choanal polyp of sphenoidal origin. Eur Arch Otorhinolaryngol 257: 379-381.

16. Soh KB, Tan KK (2000) Sphenochoanal polyps in Singapore: diagnosis and current management. Singapore Med J 41: 184-187. 